Documentation et bibliothèques

DOCUMENTATION BIBLIOTHEQUES

\title{
Les conditions de travail des bibliothécaires au Québec : étude comparative
}

\section{Louise Beauregard et Guy Dionne}

Volume 24, numéro 1, mars 1978

URI : https://id.erudit.org/iderudit/1055179ar

DOI : https://doi.org/10.7202/1055179ar

Aller au sommaire du numéro

Éditeur(s)

Association pour l'avancement des sciences et des techniques de la

documentation (ASTED)

ISSN

0315-2340 (imprimé)

2291-8949 (numérique)

Découvrir la revue

Citer cet article

Beauregard, L. \& Dionne, G. (1978). Les conditions de travail des bibliothécaires au Québec : étude comparative. Documentation et bibliothèques, 24(1), 11-22. https://doi.org/10.7202/1055179ar
Résumé de l'article

Cette étude se veut une analyse de quelques-unes des clauses des conventions collectives et des ententes qui régissent la pratique et la responsabilité professionnelles de la majorité des bibliothécaires du Québec. Elle permet de réaliser que le salaire accordé à un employé n'est pas le seul critère à considérer lors de négociations collectives ou individuelles avec un employeur. Est également inclus un bref historique de la syndicalisation des professionnels au Québec dans les secteurs publics et para-publics.
Tous droits réservés (c) Association pour l'avancement des sciences et des techniques de la documentation (ASTED), 1978
Ce document est protégé par la loi sur le droit d'auteur. L'utilisation des services d'Érudit (y compris la reproduction) est assujettie à sa politique d'utilisation que vous pouvez consulter en ligne.

https://apropos.erudit.org/fr/usagers/politique-dutilisation/ 


\section{Les conditions de travail des bibliothécaires au Québec: étude comparative}

\author{
Louise Beauregard \\ Collège de Maisonneuve \\ Montréal
}

\author{
Guy Dionne \\ Bibliothèque nationale du Québec \\ Montréal
}

Cette étude se veut une analyse de quelques-unes des clauses des conventions collectives et des ententes qui régissent la pratique et la responsabilité professionnelles de la majorité des bibliothécaires du Québec. Elle permet de réaliser que le salaire accordé à un employé n'est pas le seul critère à considérer lors de négociations collectives ou individuelles avec un employeur. Est également inclus un bref historique de la syndicalisation des professionnels au Québec dans les secteurs publics et para-publics.

This sudy is meant as an analysis of a few of the clauses of the union and other agreements which control the practice and the professional responsibility of the majority of librarians in Québec. It would seem to indicate that the salary paid an employee is not the only criteria to be considered in individual or collective negotiations with an employer. The article also contains a brief history of the unionisation of Québec professionals of the public and parapublic sectors.

Este estudio es un análisis de unas de las cláusulas de los convenios colectivos y acuerdos que rigen la práctica y la responsabilidad profesionales de la maoyria de los bibliotecarios de Québec. Vemos que el salario de un empleado no es el único criterio que se debe considerar en negociaciones colectivas o individuales con un empleador. El análisis también incluye una reseña histórica de la sindicalización de los profesionales de Québec en los sectores públicos y parapúblicos.

A la suite des négociations dans le secteur public et para-public qui, pour la majorité, se sont terminées en 1976, nous avons senti le besoin de dresser un bilan des conditions de travail des bibliothécaires syndiqués. Nous avons donc analysé et comparé les diverses conventions de ces secteurs (Fonction publique, cégeps, commissions scolaires, Univer-

1. Les conventions collectives et ententes suivantes sont celles qui ont servi à notre étude.

Convention collective intervenue entre le Gouvernement du Québec et le Syndicat des professionnels du gouvernement du Québec, 1975-1979. (1976).

Protocole entre l'Université Laval et l'Association du personnel administratif et professionnel de l'Université Laval. Inc., 1975-1978. (1976).

Protocole entre l'Université de Sherbrooke et l'Association du personnel administratif et professionnel de I'Université de Sherbrooke, Inc., 1975-1978. (1976).

Convention collective entre l'Université de Montréal sité du Québec à Montréal, Université de Montréal, Bibliothèque de la ville de Montréal et Bibliothèque municipale de Saint-Léonard), les ententes intervenues à l'Université Laval et à I'Université de Sherbrooke ainsi que la situation dans un organisme où il n'y a ni convention ni entente, la Centrale des bibliothèques' ${ }^{1}$.

et le Syndicat national des employés de l'Université de Montréal (CSN), 1975-1979. (Montréal, 1977).

Convention collective entre l'Université du Québec à Montréal et le SEUQUAM, Syndicats des employés de l'Université du Québec à Montréal, section locale 1294 (SCFP, FTQ) (1975-1979). Montréal, Université du Québec à Montréal, 1977.

Convention collective faisant suite à l'entente intervenue entre d'une part le Gouvernement du Québec et la Fédération des cégeps pour le compte des collèges d'enseignement général et professionnel et d'autre part la Fédération des professionnels salariés et des cadres du Québec (CSN) (...) 1975-1979. (Septembre 1976).

Convention collective faisant suite à l'entente inter- 
Cependant, il nous a semblé qu'il ne fallait pas nous limiter à comparer les échelles de salaire pour évaluer l'ensemble des conditions de travail des bibliothécaires dans ces divers milieux. Nous avons donc étudié une série d'aspects trop souvent négligés, comme le type de carrière et d'avancement, les possibilités de perfectionnement, la pratique et la responsabilité professionnelles, les horaires de travail et les congés avec ou sans traitement pour activités professionnelles.

Afin de permettre une meilleure appréciation des données recueillies, il importait de préciser certaines notions de base comme les types de regroupement possibles pour des professionnels face à leur employeur, les genres de carrière disponibles et, à l'intérieur, la classification, les corps d'emploi, etc. Nous avons donc abordé ces questions dans la perspective d'un historique de la reconnaissance des droits des bibliothécaires qui se confond en grande partie avec celle des professionnels salariés au Québec.

\footnotetext{
venue entre, d'une part le Gouvernement du Québec et la Fédération des CÉGEPS pour le compte des collèges d'enseignement général et professionnel et d'autre part, la Centrale de l'enseignement du Québec pour le compte des professionnels qu'elle représente (...) 1975-1979. (Septembre 1976).

Classification: plan de classification des emplois, l'avancement de classe, l'avancement accéléré d'échelon, personnel professionnel collèges d'enseignement général et professionnel. Québec, Fédération des cégeps (et) ministère de l'Education, juin 1976.

Entente intervenue entre d'une part, la Fédération des commissions scolaires catholiques du Québec pour le compte des commissions scolaires et commissions régionales pour catholiques du Québec et le ministre de l'Education et d'autre part, la Centrale de l'enseignement du Québec pour le compte de l'Association des professionnels non enseignants du Québec et du Syndicat de l'enseignement de la région des Mille-Isles pour les professionnels qu'il représente (...) 1975-1979. (Septembre 1976).
}

Convention collective entre la ville de Montréal et le Syndicat des employés de la ville de Montréal (SCFP-FTQ), 1er décembre 1975 au 30 novembre 1977.

Convention collective de travail entre la Cité de Saint Léonard et le Syndicat national des fonctionnaires municipaux de Saint-Léonard (CSN). 1er janvier 1977 au 31 décembre 1978. (1977).

N.B. : Pour obtenir des informations sur le personnel administratif tant de la fonction publique québécoise que des administrations décentralisées de l'Etat, voir: Alain Baccigalupo, L'administration québécoise, Paris, Berger-Levrault, 1976, p. 59-95 (Collection administrative).

\section{Historique du syndicalisme chez les professionnels salariés}

Lors des débats qui ont entouré l'adoption du Code du travail2 du Québec en 1964, la Confédération des syndicats nationaux, par l'intermédiaire de la Fédération des ingénieurs, se fit le principal défenseur de la syndicalisation des professionnels salariés. Elle appuyait en cela les efforts des ingénieurs de la ville de Montréal et de l'Hydro-Québec qui s'étaient regroupés en vertu de la Loi des syndicats professionnels qui permet à «quinze (15) personnes ou plus exerçant la même profession... de se constituer en Association ou Syndicat professionnel „3. C'était le seul type de regroupement possible parce qu'on voulait inclure des cadres des premiers niveaux de gérance, exclus du Code du travail en 1964 et qui le demeurent encore aujourd'hui.

Ce mouvement était une réaction à la prise de position des corporations professionnelles, à l'Ordre des ingénieurs en particulier, qui prétendaient pouvoir négocier les conditions de travail de leurs membres.

Ces professionnels salariés sentaient la nécessité de se regrouper face à leurs employeurs respectifs en dehors de leurs corporations qui, par définition, réunissent des patrons et des employés dans le but de défendre des intérêts professionnels et matériels et le droit du public à des services de qualité. D'ailleurs, le rapport de la commission CastonguayNepveu est venu confirmer cette distinction en précisant que le rôle primordial des corporations est la défense de l'intérêt du public 4 .

Dans la fonction publique du Québec, les ingénieurs et les autres professionnels, membres de corporations, avaient suivi l'exemple de leurs confrères de la ville de Montréal et de l'Hydro-Québec en formant six syndicats. Ceux qui n'étaient pas membres de corporations, comme les bibliothécaires, formèrent le Syndi-

2. "Code du travail", in Statuts refondus du Québec, 1964, chapitre 141, et modifications pour les années ultérieures.

3. "Lois des syndicats professionnels", in Statuts refondus du Québec, chapitre 146, art.1, et Statuts du Québec, 1965, chapitre 51, art. 1.

4. Jean-Rémi Brault, «La Corporation est-elle une corporation? "Argus, vol. 1; no 2 (janvier - février 1972 ), 15-31. 
cat interprofessionnel de la Fonction publique, devenu depuis le Syndicat des professionnels du gouvernement du Québec (SPGQ). Après de longues et dures négociations avec la "Reine", leur employeur, ils obtinrent leur reconnaissance comme agents négociateurs grâce à des arrêtés en conseil en 1965, ce qui n'empêcha pas une grève de treize semaines en 1966, afin d'obtenir l'inclusion des cadres de premier niveau de gérance que l'on retrouve maintenant dans la classe I des plans de carrière de ces professionnels. Nous reviendrons plus loin sur ces notions de plan de carrière et de plan par poste (position plan) dans la partie "Carrière et avancement ».

Il existe un autre type de regroupement chez les professionnels salariés, l'association bona fide. Dans ce cas, conformément à la Ille partie de la Loi des compagnies ${ }^{5}$, trois employés au minimum forment une association qui n'est cependant pas reconnue comme agent négociateur par le Code du travail. Par contre, si l'employeur accepte de négocier leurs conditions de travail, les parties peuvent signer une entente de bonne foi qui les lie aussi longtemps qu'une des parties ne la dénonce pas. L'inconvénient majeur de cette association est l'absence de toutes les garanties prévues dans le Code du travail surtout en période d'organisation. Parmi les conventions ou ententes analysées, deux sont de ce type, celle de l'Association du personnel administratif et professionnel de l'Université Laval, Inc. et celle de l'Association du personnel administratif et professionnel de l'Université de Sherbrooke, Inc.

5. "Loi des compagnies", in Statuts refondus du Québec, 1964, chapitre 271, Ille partie, art. 214, et Lois du Québec, 1969, chapitre 26, art. 48.
En vertu du Code du travail du Québec, un seul salarié peut former un syndicat et on lui octroie un ensemble de garanties dès qu'il est reconnu comme agent négociateur. Le principal critère est la représentativité (majorité de $50 \%$ plus 1 des membres de l'unité de négociation ). C'est le cas de la grande majorité des conventions étudiées: cégeps, commissions scolaires, UQUAM, Bibliothèque de la ville de Montréal, Bibliothèque municipale de SaintLéonard. Parmi ces syndicats, on distingue d'abord un regroupement par catégories de salariés: professionnels, techniciens, personnel de bureau, ou autres. On y retrouve les professionnels des cégeps et des commissions scolaires.

Par contre, un syndicat dit industriel regroupe plusieurs catégories de salariés. Appartiennent à ce type les bibliothécaires de la Bibliothèque de la ville de Montréal, de la Bibliothèque municipale de Saint-Léonard ${ }^{6}$, de l'Université de Montréal et de I'UQUAM. Dans le cas de la ville de Montréal, nous nous interrogeons sur le fait que les bibliothécaires soient intégrés au Syndicat des fonctionnaires municipaux alors qu'il existe un Syndicat des professionnels de la ville de Montréal. D'un autre côté, on retrouve aussi des syndicats indépendants et certains qui sont affiliés à une centrale syndicale qui regroupe des syndicats soit d'un même secteur d'activités (affaires sociales, par exemple), soit d'un même niveau de scolarité (professionnels salariés).

6. Parmi les quatre bibliothécaires à l'emploi de la Bibliothèque municipale de Saint-Léonard, une seule bibliothécaire est syndiquée. Les trois autres bibliothécaires appartiennent à la catégorie "cadres ". Les conditions de travail de ces derniers ne sont pas considérées dans cet article.

\section{Carrière et avancement}

Tableau 1

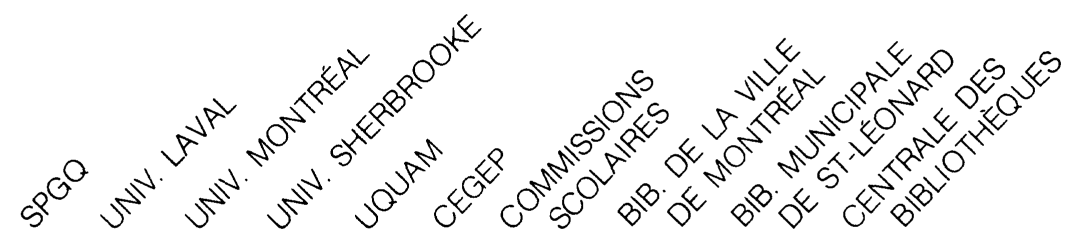

$\begin{array}{llllllll}\text { Plan de carrière (année) } & 15 & 11 & 11 & 11 & 15 & 15^{\star} & 15^{*}\end{array}$

Plan par poste

$6 \quad 6$

$15^{\star}$

* Equivaut à 14 ans pour ceux qui sont classés dans le corps d'emploi de Bibliothécaire. 
Pour l'ensemble des professionnels, le plan de carrière est plus avantageux que le plan par poste à moins d'envisager des responsabilités administratives à court terme. Le plan de carrière permet l'avancement continu sur un rendement satisfaisant jusqu'au maximum de l'échelle de salaire. Le plan par poste suppose des promotions successives; le professionnel atteint plus rapidement le maximum salarial de ce poste, mais s'il veut progresser, il doit subir un concours pour accéder à un poste supérieur comportant plus de responsabilités administratives et une échelle de salaire supérieure.

Une autre caractéristique qui rend avantageux le plan de carrière est certainement le fait qu'il est plus difficile de changer la description d'un corps d'emploi que de changer la description d'un poste. Ceci procure donc une plus grande sécurité dans l'emploi. Le professionnel, une fois qualifié pour un corps d'emploi, peut occuper différents postes rattachés à ce même corps d'emploi.

Dans le tableau I, on remarquera que les universités de Montréal, de Sherbrooke et Laval possèdent un régime mixte. Elles possèdent un plan de carrière de onze ans équivalant aux classes III et II. Au-delà de la classe II de l'échelle salariale, l'avancement se fait par promotion à un poste comportant plus de responsabilités administratives.
Les bibliothécaires sont majoritairement, à cause du SPGQ, classifiés dans le corps d'emploi de Bibliothécaire, mais dans certains secteurs comme dans les universités, les cégeps et les commissions scolaires, ils sont classifiés, depuis les dernières négociations, dans le corps d'emploi des Spécialistes en moyens et techniques d'enseignement (SMTE).

C'est chez les professionnels du gouvernement que les exigences sont les plus fortes pour le passage d'une classe à une autre ( III à II et II à I). Pour accéder à une autre classe, le professionnel doit nécessairement se présenter devant un jury sur recommandation de son supérieur. Les critères de passage sont cependant connus.

Au niveau universitaire, pour le passage de la classe II à I, comme nous l'avons déjà dit, l'accession se fait suivant la disponibilité de postes syndiqués ou non syndiqués comportant des responsabilités administratives. La majorité des cégeps ont remplacé par diverses procédures locales le recours au jury pour le passage de la classe II à I.

La permanence d'emploi s'acquiert après un an dans la majorité des cas, sauf à I'UQUAM, à la Bibliothèque de la ville de Montréal et à la Bibliothèque municipale de Saint-Léonard où elle s'acquiert après six

Tableau $\|$

Classement à l'entrée selon critères connus

Négociations personnelles Avancement d'échelon accéléré Avancement de classe III à II jury critères connus

II à I jury critères connus postes disponibles

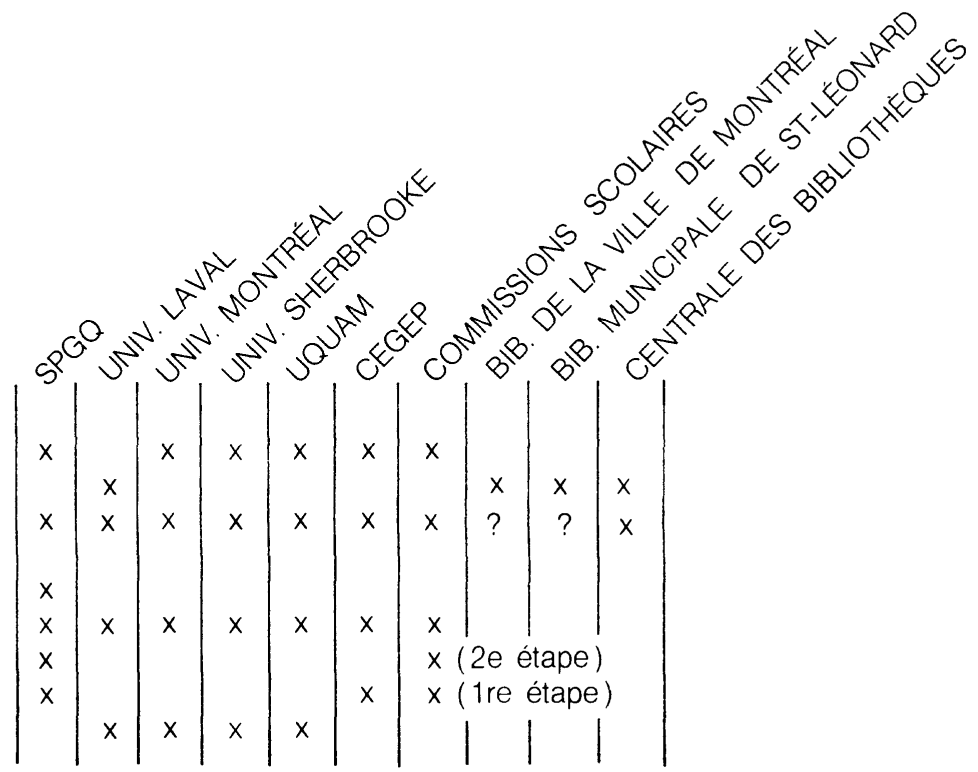


mois; I'UQUAM cependant pourra exiger douze mois dans le cas de changement technique ou administratif. Dans les cégeps, la permanence est obtenue après dix-huit mois et dans les commissions scolaires, après deux ans. La Centrale des bibliothèques n'a pas la permanence d'emploi: tant que le contrat n'est pas signé entre le gouvernement (pourvoyeur de fonds) et le Centre d'animation, de développement et de recherche en éducation (CADRE) - organisme qui administre la Centrale - le professionnel n'est pas assuré de son poste. L'engagement est renouvelable le 1er avril de chaque année alors que débute la nouvelle année financière. II y a cependant nécessité d'un avis de trois mois, c'est-à-dire que si le 31 décembre, le professionnel n'a pas reçu d'avis de non-renouvellement, son engagement est reconduit pour une autre année.

Pour ce qui est de la sécurité d'emploi, seul le SPGQ jouit d'une sécurité absolue en vertu du statut de la Fonction publique que les dispositions de la loi ont dissocié du pouvoir politique. La Bibliothèque de la ville de Montréal et celle de Saint-Léonard disposent aussi de ce type de sécurité d'emploi: l'employeur s'engage à trouver un poste à l'employé. Dans les cégeps et les commissions scolaires, la sécurité d'emploi est assurée après deux ans au niveau des réseaux respectifs. II existe cependant une priorité d'emploi dans le réseau après douze mois; il ne peut y avoir d'abolition de poste que pour des raisons bien précises stipulées dans les différentes conventions collectives. Dans les universités, il y a une sécurité d'emploi plus mitigée car, dans le cas d'abolition de poste, on peut exiger le recyclage ou la mutation; le nombre de postes disponibles est plus limité que dans les réseaux des cégeps et des commissions scolaires.

\section{Pratique et responsabilités professionnelles}

Cet aspect des conditions de travail nous semble révélateur du degré d'autonomie reconnu aux professionnels des différents secteurs. Historiquement, le SPGQ avait obtenu une reconnaissance de ce droit en 1966 pour faire échec à toute tentative d'ingérence politique dans les travaux professionnels. Par la suite, en 1973, ce même droit était reconnu aux professionnels des cégeps et des commissions scolaires lors de leurs premières négociations. ,

Aux universités de Sherbrooke, de Montréal et à l'UQUAM, le professionnel peut, s'il le désire, signer son document. Advenant le cas où il refuse de signer parce que le texte en a été modifié, en tout ou en partie, les universités concernées ne peuvent lui imposer aucune mesure disciplinaire. A l'Université Laval,
Document rédigé par le professionnel:

- propriété de l'auteur

- propriété de l'employeur

si utilisé par l'employeur '

la signature de l'auteur doit apparaître

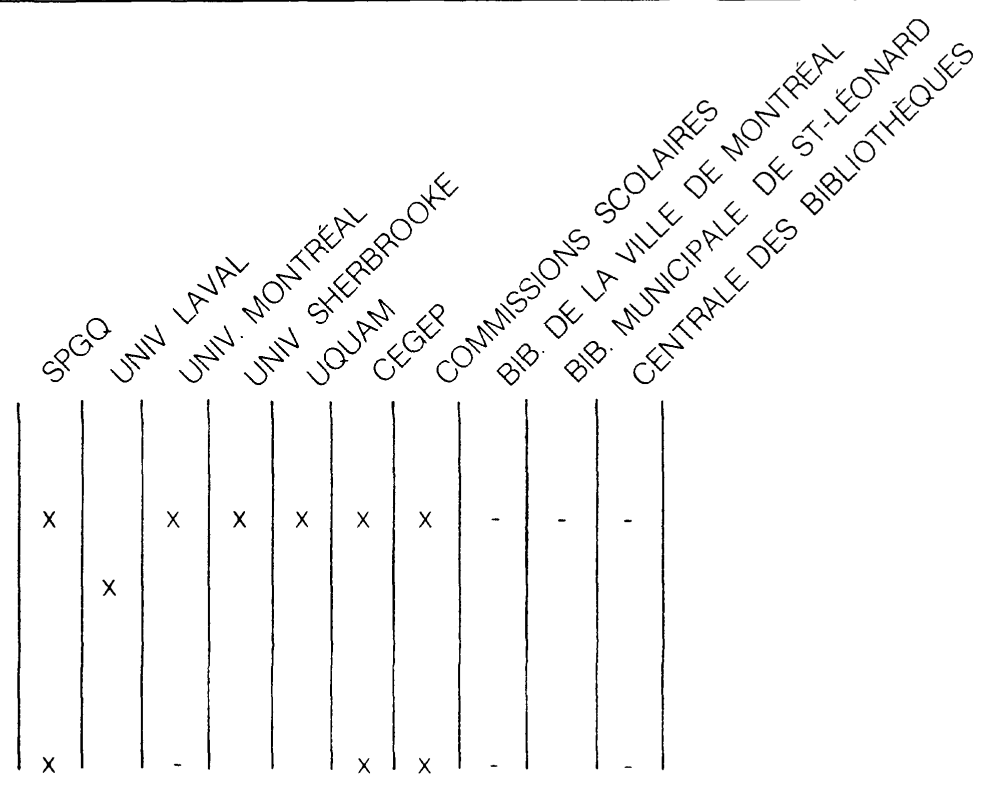


Tableau IV

Provincial

Régional

Local

Comité de perfectionnement

paritaire

consultatif

- décisionnel
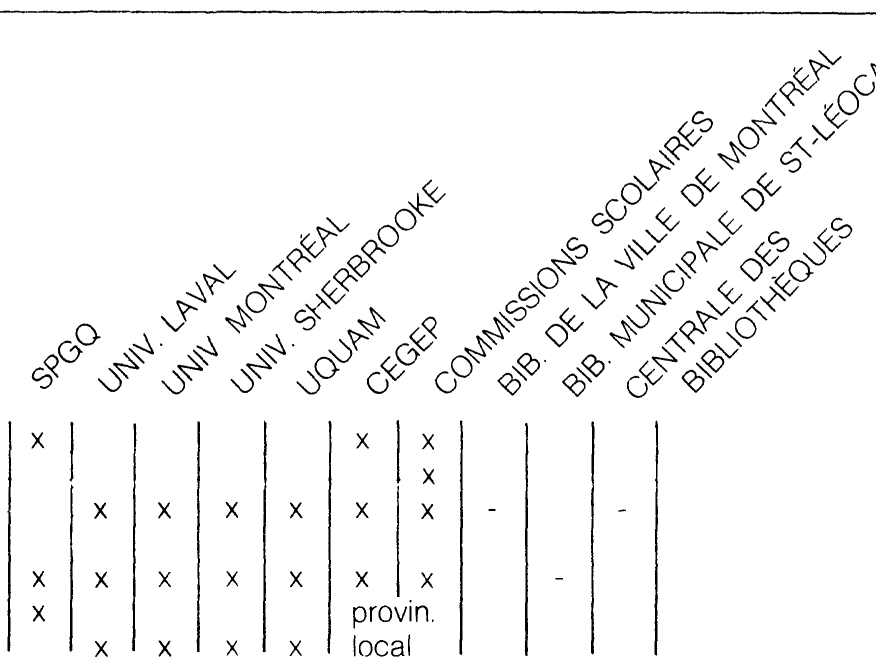

«|'Université est propriétaire de tout produit... » (article 46).

A la Centrale des bibliothèques, comme il n'y a ni entente écrite ni convention collective, il est difficile d'en juger. En pratique, on constate que des bibliographies et certains textes ou études sont signés par leurs auteurs.

Pour ce qui est de la Bibliothèque de la ville de Montréal et de la Bibliothèque municipale de Saint-Léonard, il n'en est fait mention nulle part. En pratique, à la Bibliothèque de la ville de Montréal en particulier, selon les différents services, l'autonomie professionnelle est plus ou moins reconnue. Fait à remarquer, les syndicats qui regroupent les bibliothécaires dans ces deux bibliothèques sont des syndicats de type industriel.

\section{Perfectionnement}

Aux universités Laval, de Montréal et de Sherbrooke, selon certaines modalités, la tradition veut que le professionnel puisse suivre gratuitement des cours de perfectionnement dans l'institution qui l'emploie. Dans les commissions scolaires, les cégeps et à I'UQUAM, le professionnel peut aussi s'inscrire gratuitement aux cours que dispense l'organisme employeur; cela apparaît dans les conventions.

A la Centrale des bibliothèques, I'horaire flexible en vigueur permet à un professionnel de poursuivre des études de perfectionnement qui ne sont pas nécessairement reconnues au niveau de la rémunération.
A la Bibliothèque de la ville de Montréal et à la Bibliothèque municipale de Saint-Léonard, on n'encourage ni ne décourage personne à se perfectionner. Chacun s'organise pour aménager son temps de travail, en fonction de ses cours, avec ses compagnons de travail; on peut aussi négocier personnellement avec son employeur les modalités de perfectionnement qui ne peuvent être que coupure salariale ou remise du temps. La Bibliothèque de la ville de Montréal, sur présentation d'une preuve de réussite, peut rembourser la moitié des frais de scolarité. A la Bibliothèque municipale de Saint-Léonard, si l'employé poursuit un cours dont le contenu est en relation directe ou indirecte avec son travail, l'employeur paie les frais de ce cours et l'employé doit remettre son temps ou assumer une perte salariale.

A l'UQUAM, dans les cégeps, les commissions scolaires et au SPGQ, il existe des politiques de perfectionnement similaires qui ont été négociées.

A l'UQUAM, on dispose d'un budget réservé au perfectionnement dont l'utilisation s'effectue selon les décisions du Comité paritaire de perfectionnement.

Dans les cégeps, il existe deux types de perfectionnement: provincial et local. Au niveau provincial, une somme indexée $(\$ 247,000$ pour 1978-1979) est à la disposition des professionnels. Le Comité consultatif paritaire de perfectionnement des professionnels (CCPPP) regroupe des représentants du mi- 
nistère de l'Education, des commissions scolaires et des cégeps, d'une part, et des représentants des différents syndicats de professionnels oeuvrant dans les commissions scolaires et les cégeps, d'autre part. Le plus souvent, les cours sont offerts par les diverses constituantes de l'Université du Québec. Au niveau local, une somme de $\$ 30.00$ pour l'année 1977-1978 (\$31.00 pour 1978-1979) par professionnel est versée au cégep. Cette somme est administrée par un comité paritaire local de perfectionnement qui, lui, est décisionnel.

Dans les commissions scolaires, il existe les types provincial et local décrits ci-dessus; il y a aussi des possibilités de regroupement régional. Pour un projet spécial au niveau régional, on peut demander une certaine somme provenant du fonds provincial ou mettre en commun les budgets prévus au niveau local.

Au SPGQ, il existe un Programme de développement planifié des ressources humaines auquel le Syndicat participe de façon consultative par l'entremise du Comité des relations professionnelles. La détermination du budget de perfectionnement se fait annuellement au niveau ministériel en consultation avec le Comité ministériel des relations professionnelles.

\section{Activités professionnelles, congé sans solde}

Ces activités sont connexes au perfectionnement et nous considérons qu'elles permettent aussi d'évaluer le degré d'autonomie professionnelle.

Les professionnels des secteurs cégeps, commissions scolaires et SPGQ peuvent bénéficier d'un congé avec solde pour donner des cours et des conférences et pour participer à des séminaires, congrès ou colloques. A la Bibliothèque de la ville de Montréal, la participation est limitée à quelques personnes. A la Centrale des bibliothèques, tout est fonction de l'horaire flexible. Dans les universités, sauf à I'UQUAM, selon le bon vouloir de l'employeur le professionnel peut assister à des congrès ou des colloques tout en conservant son salaire. A I'UQUAM, en cas de refus de l'employeur, le professionnel a un droit de grief.

Quant aux congés sans solde, dont nous excluons les congés pour maternité, ils peu- vent être accordés pour un an au moins dans tous les secteurs, sauf à la Centrale des bibliothèques. A la Bibliothèque municipale de Saint-Léonard, il existerait cependant une politique locale qui permettrait le congé sans solde. A la Bibliothèque de la ville de Montréal. il existe des congés sans solde pour lesquels cependant les motifs d'étude ou de perfectionnement ne sont pas jugés valables.

Dans les secteurs qui offrent la possibilité des congés sans solde. l'employeur juge de la valeur du motif invoqué par le professionnel. En cas de refus, le professionnel du SPGQ. de l'Université de Montréal et de I'UQUAM a un droit de grief. Dans le secteur cégep, le professionnel n'a pas à fournir de motif lors de sa demande et la seule restriction est celle "de quitter de facon temporaire son poste pour occuper un autre emploi à mois que celui-ci puisse être considéré comme du recyclage " (article 5-13.06).

On offre la garantie du même poste ou d'un poste équivalent au retour du professionnel. sauf dans les cégeps où le poste est assuré Autre particularité du secteur cégep : le professionnel peut demander, aux conditions décrites ci-dessus, un congé mi-temps, mi-traitement.

\section{Para-monétaire}

A la Bibliothèque municipale de SaintLéonard, le personnel ne travaille que ${ }^{11}$ heures 10 minutes par semaine.

Dans la majorité des secteurs, les professionnels travaillent 32 heures et demie par semaine. Le SPGQ et I'Université de Sherbrooke ont inscrit dans leurs conventions collectives ce nombre d'heures et, dans la presque totalité des cégeps et des commissions scolaires, on pratique la semaine de 32 heures et demie, bien que le nombre de 35 heures soit inscrit dans les conventions.

A la Bibliothèque de la ville de Montréal, les bibliothécaires travaillent 33 heures et trois quarts par semaine. Mais aux services publics, le bibliothécaire doit, en alternance, effectuer une semaine de 40 heures et demie répartie du lundi au samedi et une semaine de 27 heures répartie du mardi au vendredi. De plus, ce bibliothécaire travaille, soit l'après-midi, soit 
Tableau $V$

Horaire

- 35 heures

35 heures

+35 heures

flexible

variable

d'été avec diminution des heures

Temps supplémentaire en sus de 40 heures en sus de 35 heures remboursement en temps argent

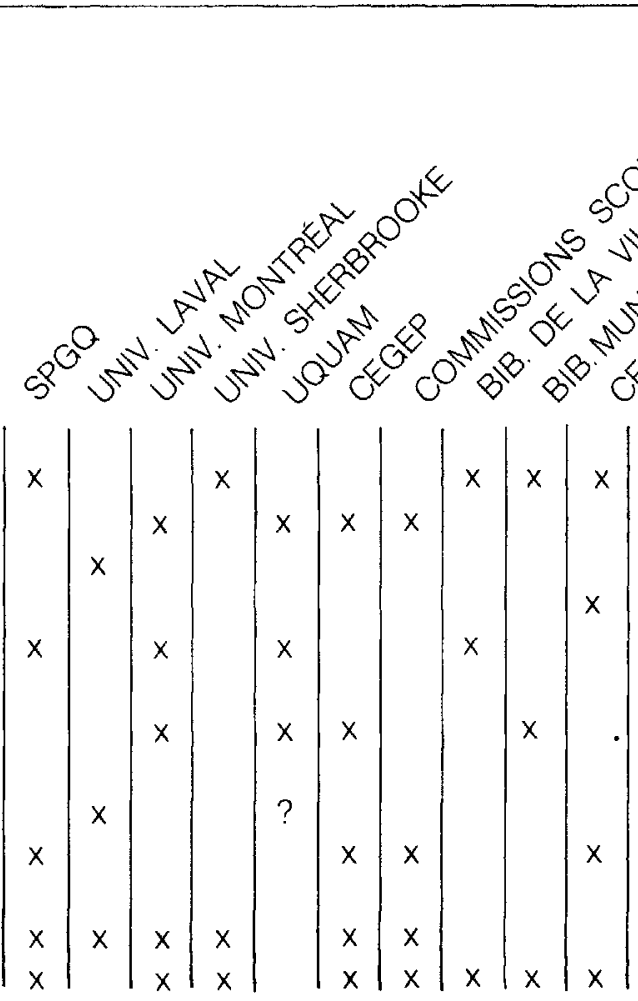

l'avant-midi et le soir. Dans ce dernier cas, l'horaire fractionné entraine des déboursés supplémentaires au niveau du transport car il implique deux aller-retour au domicile. 11 existe une prime de $15 \%$ du taux horaire pour le travail effectué après 16 heures trente et le samedi. A la Bibliothèque municipale de SaintLéonard, les employés ont une échelle de salaire majorée de $5 \%$ en compensation du travail effectué selon un système de rotation

Tableau $\mathrm{VI}$

\section{Vacances}

après un an

10 jours

20 jours

1 mois (21 ou 22 jours) après 2 ans: 3 semaines après 3 ans: 3 semaines après 5 ans: 4 semaines après 15 ans: 4 semaines

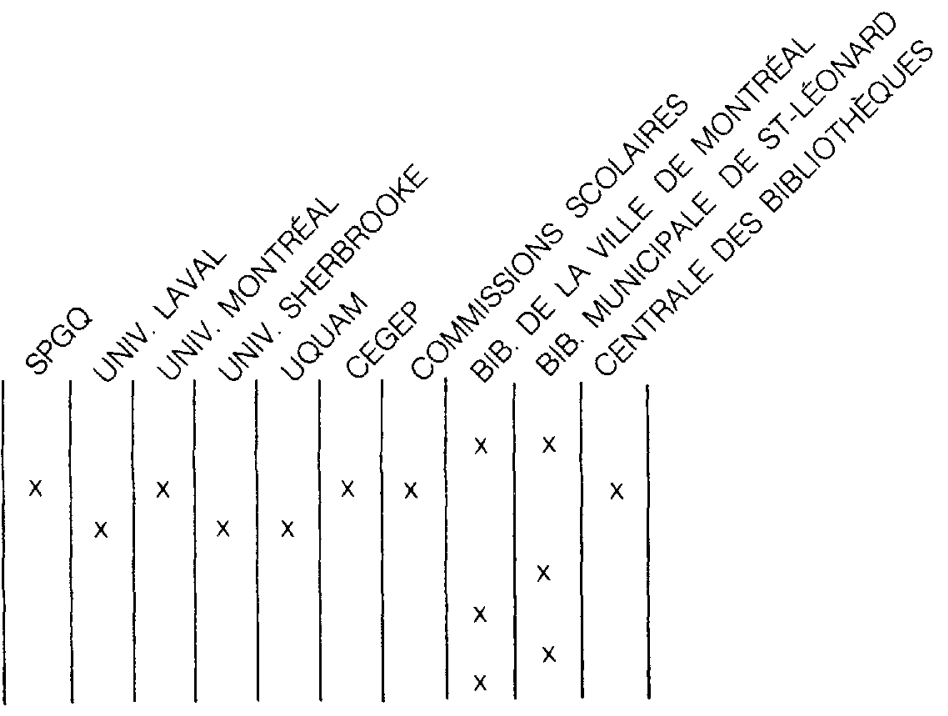


des heures les obligeant à travailler certains soirs ou en fin de semaine.

Lorsqu'il effectue du temps supplémentaire, le professionnel est remboursé au taux simple en temps ou en argent, sauf à la Bibliothèque municipale de Saint-Léonard et à la Bibliothèque de la ville de Montréal où la personne est remboursée à taux et demi et à taux double dans certains cas.

Dans les secteurs public et para-public, le professionnel, après 17 et 18 ans d'expérience, a droit à 21 jours de vacances augmentant ainsi d'une journée à tous les deux ans d'expérience supplémentaire jusqu'à concurrence de 25 jours pour 25 ans d'expérience et plus.

On remarque aussi que les moins bien favorisés sont ceux qui travaillent à la Bibliothèque de la ville de Montréal et à la Bibliothèque municipale de Saint-Léonard. Serait-ce encore le fait que ces bibliothécaires font partie d'un syndicat de type industriel?

\section{Rémunération}

Quoique nous pensions que le chapitre des conventions portant sur la rémunération ne soit pas le plus important, il reste que, pour la majorité des gens, c'est le plus remarqué et le plus discuté.

Les bibliothécaires ont eu raison, lors de la négociation des conventions précédentes, de se préoccuper énormément de la question des échelles de salaire. Leur échelle était vraiment discriminatoire par rapport aux échelles de salaire des autres professionnels. Dans les conventions ou ententes signées en 1976 et 1977, les administrations ont accepté de réviser les échelles à cause des pressions exercées.

La Fonction publique a conçu l'échelle du corps d'emploi de Bibliothécaire selon les mêmes éléments que les autres échelles, c'est-à-dire qu'elle a prévu le même nombre d'échelons dans chaque classe et des échelons semi-annuels dans la classe III.

Les bibliothécaires du SPGQ sont toujours intégrés à l'échelle du corps d'emploi de Bibliothécaire. Quelques-uns sont cependant classés dans les corps d'Agent culturel, Agent d'information, Agent de recherche, Attaché d'administration, ou autres et dans les échelles de salaire correspondant à leur corps respectif.

Dans les secteurs des universités, cégeps, commissions scolaires et à la Centrale des bibliothèques, l'échelle des Bibliothécaires apparait dans les conventions, mais les bibliothécaires ont presque tous accédé à l'échelle du corps d'emploi Spécialiste en moyens et techniques d'enseignement. La Centrale des bibliothèques intègre quelques bibliothécaires dans l'échelle du corps Spécialiste en sciences de l'éducation.

Les bibliothécaires des universités Laval et de Sherbrooke ont obtenu l'échelle la plus élevée, c'est-à-dire celle qui correspond au corps d'emploi Psychologue on Ingénieur dans la Fonction publique. II faut se souvenir cependant que la classe I de l'échelle ne peut être atteinte que par un petit nombre de bibliothécaires (cf. "Carrière et avancement »).

Les seuls bibliothécaires qui ne jouissent pas d'échelles de salaire indexées au coût de la vie sont ceux de la Bibliothèque de la ville de Montréal et de la Bibliothèque municipale de Saint-Léonard. Ce sont les mêmes qui, somme toute, possèdent les plus basses échelles de traitement. Les échelles de salaire sont cependant négociées annuellement à la Bibliothèque de la ville de Montréal. A la Bibliothèque municipale de Saint-Léonard, la durée de la convention collective est de deux ans: on y prévoit des échelles de salaire pour la première année et d'autres échelles pour la deuxième année de la convention.

Voici donc les différentes échelles de traitement dans lesquelles sont intégrés les bibliothécaires selon les lieux de travail.

Au SPGQ, dans certaines commissions scolaires et dans quelques cégeps, on a conservé l'échelle du corps d'emploi Bibliothécaire ( voir tableau VII). On reconnaît toutefois deux ans d'expérience lorsque le bibliothécaire a obtenu sa maitrise en bibliothéconomie étant donné que la qualification minimale requise est celle d'un baccalauréat. Les échelons de la classe III sont des échelons semiannuels. Cette échelle va du 1er juillet 1977 au 30 juin 1978. 
Tableau VII

$\begin{array}{cll}\text { Classe III, échelon } & 1 & 12,389 \\ & 2 & 12,900 \\ 3 & 13,428 \\ & 4 & 13,980 \\ 5 & 14,551 \\ & 6 & 15,146 \\ & 7 & 15,766^{*}\end{array}$

( ${ }^{*}$ cet échelon existe en cas de refus de passage à la classe II)

$\begin{array}{lll}\text { Classe II. échelon } & 1 & 16,414 \\ & 2 & 17,087 \\ 3 & 17,788 \\ 4 & 18,516 \\ & 5 & 19,275 \\ & 6 & 20,065^{\star} \\ & 7 & 21,887 \\ & 8 & 21,743\end{array}$

( ${ }^{*}$ dès cet échelon, il y a possibilité de passage à la classe 1)

$\begin{array}{lll}\text { Classe I, échelon } & 1 & 21,309 \\ & 2 & 22,014 \\ 3 & 22,739 \\ & 4 & 23,490 \\ & 5 & 24,264\end{array}$

La majorité des commissions scolaires et des cégeps, l'Université de Montréal, I'UQUAM et la Centrale des bibliothèques accordent l'échelle de traitement du corps d'emploi Spécialiste en moyens et techniques d'enseignement (voir tableau VIII).

Comme pour l'échelle de salaire du corps d'emploi Bibliothécaire, les commissions scolaires et les cégeps accordent toujours deux ans d'expérience supplémentaire à un bibliothécaire qui possède une maitrise. L'échelon 5 de la classe III est donc l'échelon du bibliothécaire sans expérience, ce qui équivaut à $\$ 15,274$ par année. II faut se rappeler que la classe III possède des échelons semi-annuels. A la Centrale des bibliothèques, c'est sensiblement le même processus; ce n'est cependant pas écrit. II n'est pas toujours certain que les professionnels se fassent reconnaitre toute leur scolarité ou toutes leurs années d'expérience. A l'Université de Montréal et à I'UQUAM, la classe III n'a que quatre échelons annuels et on n'y reconnaît qu'une seule année d'expérience pour la maîtrise. De plus, la classe I n'est pas accessible à tous, car elle est réservée à des postes administratifs.

L'échelle qui suit va du 1er juillet 1977 au 30 juin 1978.

Tableau VIII

$\begin{array}{ccccr}\text { Classe III, échelon } & \begin{array}{c}\text { Commissions } \\ \text { scolaires } \\ \text { Cégeps }\end{array} & \text { Universités } \\ & 1 & 13005 & 1 & 13005 \\ & 2 & 13537 & 2 & 14094 \\ & 3 & 14094 & 3 & 15274 \\ 4 & 14671 & 4 & 16551 \\ & 5 & 15274 & & \\ 6 & 15898 & & \\ & 7 & 16551^{*} & \end{array}$

( ${ }^{*}$ existe en cas de refus de passage à la classe II)

$\begin{array}{ccc}\text { Classe II, échelon } & 1 & 17568 \\ 2 & 18300 \\ 3 & 19062 \\ 4 & 19855 \\ 5 & 20683 \\ 6 & 21543^{*} \\ 7 & 22439 \\ 8 & 23373\end{array}$

( ${ }^{*}$ échelon de passage à la classe 1)

$\begin{array}{lll}\text { Classe I, échelon } & 1 & 23330 \\ & 2 & 24338 \\ 3 & 25389 \\ 4 & 26487 \\ 5 & 27630 \\ 6 & 28824\end{array}$

Les universités Laval et de Sherbrooke offrent l'échelle de salaire la plus élevée aux bibliothécaires (voir tableau IX). Par contre, l'Université de Sherbrooke ne reconnait qu'une seule année d'expérience à ceux qui possèdent une maitrise. Nous n'avons pu vérifier ce que l'Université Laval accorde à ce sujet, on n'en fait mention nulle part dans le protocole d'entente; I'Université s'engage à respecter cinq normes, mais celles-ci ne proposent aucune reconnaissance de cette scolarité. De plus, la classe I n'est pas accessible à tous. 
Cette échelle couvre la période du 1er juin 1977 au 30 mai 1978.

Tableau IX

$\begin{array}{lll}\text { Classe III. } \quad \text { échelon } & 1 & 13563 \\ & 2 & 14157 \\ 3 & 14781 \\ 4 & 15434 \\ & 5 & 16113 \\ 6 & 16821 \\ & 7 & 17566 \\ & & \\ \text { Classe II, échelon } & 1 & 18721 \\ & 2 & 19539 \\ & 3 & 20394 \\ & 4 & 21285 \\ & 5 & 22215 \\ & 6 & 23184^{*} \\ & 7 & 24198 \\ & 8 & 25255\end{array}$

( ${ }^{*}$ échelon de passage à la classe I si un poste est disponible).

$\begin{array}{rrr}\text { Classe I, échelon } & 1 & 25093 \\ & 2 & 25970 \\ 3 & 26880 \\ 4 & 27821 \\ & 5 & 28794 \\ 6 & 29802\end{array}$

Les trois échelles de salaire précédentes sont indexées au coût de la vie, c'est-à-dire que, si entre juin 1977 et juin 1978, il y a une augmentation de $8 \%$ du coût de la vie, les échelles pour la période de juin ou juillet 1978 à mai ou juin 1979 demeureront les mêmes mais seront majorées de $8 \%$.

A la Bibliothèque de la ville de Montréal, l'échelle de traitement qui se terminait le 30 novembre 1977 reste toujours en vigueur jusqu'à l'acceptation d'une nouvelle échelle présentement en négociation. II ne faut pas oublier que, parce que les bibliothécaires possèdent un plan par poste, ils ont une échelle réduite à six échelons dont le minimum, actuellement, est $\$ 12,478$ et le maximum $\$ 15,942$. II est prévu, entre le minimum et le maximum, une augmentation statutaire de $\$ 760$ annuellement.

A la Bibliothèque municipale de SaintLéonard, le même type d'échelle s'applique et cette dernière est en vigueur depuis le ler janvier 1978. La classe 6 des employés de Saint-Léonard comporte six échelons dont le minimum est $\$ 14,129$ et le maximum $\$ 17,537$. L'augmentation statutaire entre les échelons est de $\$ 568$ annuellement.

Ces deux dernières échelles de traitement, comme nous l'avons dit précédemment. ne sont pas indexées au coût de la vie: elles sont cependant négociables annuellement ou, comme à la Bibliothèque municipale de SaintLéonard, une échelle de salaire est prévue pour chacune des deux années de la durée de la convention collective.

\section{Conclusion}

Au terme de ce survol des conditions de. travail des bibliothécaires, il ressort clairement que le secteur syndiqué le plus défavorisé est celui des bibliothèques publiques. Cette situation ne contribue certes pas à attirer de nouveaux professionnels dynamiques. II semble toutefois qu'à la Bibliothèque de la ville de Montréal une restructuration administrative majeure soit déjà amorcée et qu'elle permettra de créer un meilleur climat professionnel. On prévoit, entre autres choses, une plus grande décentralisation laissant une plus large autonomie au professionnel ainsi qu'une révision des horaires de travail principalement pour les bibliothécaires des services au public. La présente négociation salariale donnera peutêtre lieu à une meilleure reconnaissance $d u$ caractère professionnel des bibliothécaires par l'attribution d'un niveau de salaire supérieur.

Quant à la Centrale des bibliothèques, vu l'absence de toute sécurité d'emploi et devant le règne de l'arbitraire principalement au plan de la reconnaissance de la scolarité et de l'expérience, nous ne pouvons que souhaiter la formation d'un syndicat le plus rapidement possible. Certains diront que les bibliothécaires y sont bien payés. En effet, comme nous l'avons déjà dit, l'échelle de salaire est celle du Spécialiste en moyens et techniques d'enseignement ou celle du Spécialiste en sciences de l'éducation, mais l'interprétation, en pratique, diffère des autres secteurs qui jouissent des mêmes échelles. Au risque de nous répéter, rappelons encore que le traitement qu'on accorde à un employé n'est pas le seul aspect à considérer lors du choix d'un emploi. 
Bien que l'ensemble des conditions de travail soit satisfaisant et que la tendance soit à l'uniformisation des échelles de salaire, les bibliothécaires du SPGQ espèrent avoir accès aux échelles de salaire des secteurs des cégeps et des commissions scolaires. Le plan de classification n'est cependant pas négociable puisqu'il est régi par la Commission de la Fonction publique qui n'est pas une des parties lors d'une négociation.

Dans les universités de Sherbrooke, Laval, de Montréal et à l'UQUAM, il faudra reconnaître un véritable plan de carrière aux bibliothécaires, en leur donnant accès à la classe I sans exiger l'occupation d'un poste comportant des responsabilités administratives. II serait souhaitable que les bibliothécaires des universités obtiennent, lors des prochaines négociations, de meilleures conditions d'exercice de leur profession. Pour les bibliothécaires des universités Laval et de Sherbrooke, il apparaît qu'une association bona fide laisse beaucoup de domaines importants à l'arbitraire de l'employeur.

Les conditions de travail dans les commissions scolaires, et plus particulièrement dans les cégeps, nous semblent les plus intéressantes tant à cause du salaire que du contexte professionnel. L'interprétation des différentes conventions est laissée à chaque administration; on retrouve donc, dans ces deux secteurs, des applications plus ou moins souples des diverses clauses des conventions.

Nous espérons que cette étude comparative des principales conditions d'emploi des bibliothécaires servira à sensibiliser un plus grand nombre d'entre nous à différents aspects interreliés d'une convention ou d'une entente. Nous pensons aussi faciliter la tâche des finissants de l'Ecole de bibliothéconomie en leur fournissant des éléments comparatifs tirés des divers milieux de travail.

Lors des prochaines négociations, nous croyons que l'accent ne sera pas mis sur les conditions salariales. II faudra considérer beaucoup plus les conditions de la pratique et de la responsabilité professionnelle, l'amélioration des plans de classification et les différents avantages sociaux, telles les assurances vie, maladie et salaire. De même, le perfectionnement des professionnels prendra une importance plus considérable puisque le développement rapide des connaissances et des techni- ' ques nécessitera un recyclage périodique. 